\title{
Erratum to: Basic loci of Coxeter type in Shimura varieties
}

\author{
UlRICH GÖRTZ* AND XUhuA HE ${ }^{\dagger}$
}

We correct a few mistakes in [1].

The main results remain unaffected (but the "summary" in Theorem B in the introduction of loc. cit. needs to be corrected).

AMS 2000 SUBJECT CLASSIFICATIONS: 11G18, 14G35, $20 \mathrm{G} 25$.

KEYwORDS AND PHRASES: Reduction of Shimura varieties, affine DeligneLusztig varieties, Newton stratification.

\section{Overview}

We begin with an overview of the required changes. The statement of [1] Thm. B should be corrected as follows:

Theorem B. If $(G, \mu, P)$ is of Coxeter type, then

(1) The basic locus decomposes as

$$
X(\mu, b)_{P}=\sqcup_{\mathbb{J}_{b} /\left(\mathbb{J}_{b} \cap \operatorname{Ker} \kappa_{G}\right)} \sqcup_{\Lambda} \mathcal{N}_{\Lambda}^{o},
$$

where $\kappa_{G}$ is the Kottwitz homomorphism for the group $G$ and where $\Lambda$ runs over faces of certain types of the rational Bruhat-Tits building $\mathscr{B}$ of the $\sigma$-centralizer $\mathbb{J}_{b}$ of $b$, and $\mathcal{N}_{\Lambda}^{o}$ is a classical Deligne-Lusztig set associated to a twisted Coxeter element.

(2) For all non-basic $b^{\prime}$, the action of the $\sigma$-centralizer $\mathbb{J}_{b^{\prime}}$ on $X\left(\mu, b^{\prime}\right)_{P}$ has only finitely many orbits. In particular, $\operatorname{dim} X\left(\mu, b^{\prime}\right)_{P}=0$.

There are two changes in comparison to the original version:

Part (1). The union must be split up according to $\mathbb{J}_{b} /\left(\mathbb{J}_{b} \cap \operatorname{Ker}\left(\kappa_{G}\right)\right)$. This is also important for the description of the closure relations between strata: An analogous correction needs to be made in Prop. 7.2.2. See Section 3 below.

${ }^{*}$ U.G. was partially supported by the Sonderforschungsbereich/Transregio 45 of the DFG.

${ }^{\dagger}$ X.H. was partially supported by NSF DMS-1463852. 
Part (2). In loc. cit., it was claimed that the $\mathbb{J}_{b^{\prime}}$-action is transitive in this situation. This is not true in general. In fact, as stated in Thm. 5.2.1 (2), in the non-basic case, the action of $\mathbb{J}_{b^{\prime}}$ on $X_{J, w}\left(b^{\prime}\right)$ is transitive, so the number of orbits is just the number of elements in $\mathrm{EO}^{J}(\mu)$ with Newton vector equal to the Newton vector of $b^{\prime}$. These Newton vectors are listed in each of the cases in loc. cit., Section 6. There are 2 cases (in 6.4 and 6.6) where two different non-basic EO strata have the same Newton vector, in which case we have two orbits. In all other cases, the action is in fact transitive.

It turns out that in the classification in loc. cit., Section 6 , one case is missing: This case comes from the affine Dynkin type $\tilde{A}_{1}$ and arises from a unitary group $U(3)$ splitting over a ramified extension. Note that the list in Thm. 5.1.2 is correct and contains this case as $\left(C-B C_{1}, \omega_{1}^{\vee}, \mathbb{S}\right)$, $\left(C-B C_{1}, \omega_{1}^{\vee},\{0\}\right)$ (these two cases differ only with respect to the orientation of the affine Dynkin diagram which is ignored in Section 6). It also occurs, as it should, in [2], as the case $\left(\tilde{A}_{n-1}, \omega_{1}^{\vee}+\omega_{n-1}^{\vee}\right)$ for $n=2$. See Section 2 below for details.

Finally, it turns out that the list in Prop. 7.3.2 is not complete, and the more general statement given below is actually simpler. See the discussion in Section 4 below.

Acknowledgments. We are grateful to Haifeng $\mathrm{Wu}$ for several discussions which led to the discovery of some of the problems discussed here.

\section{Classification}

In [1] Section 6.2, in the list in the second paragraph on p. 341, we list the pairs $(\tilde{W}, \lambda)$ coming from pairs $(G, \mu)$ where $G$ is absolutely quasi-simple and $\mu$ is minuscule. However, the case $\left(\tilde{A}_{1}, 2 \omega_{1}^{\vee}\right)$ is missing from this list; it arises from a unitary group $U(3)$ splitting over a ramified quadratic extension. (For higher rank odd ramified unitary groups, we get affine Dynkin type $\tilde{B}_{n}$.)

This case was then also overlooked when checking the Coxeter type condition in Section 6.3. In fact, for type $\tilde{A}_{1}, \mu=2 \omega_{1}^{\vee}, J=\mathbb{S}$ and $\sigma=\mathrm{id}$, we get $\mathrm{EO}_{\sigma, \mathrm{cox}}^{J}=\left\{\tau, s_{0} \tau\right\}$, and $\mathrm{EO}^{J}(\mu)-\mathrm{EO}_{\sigma, \mathrm{cox}}^{J}=\left\{s_{0} s_{1} \tau\right\}$ consists of the $\sigma$-straight element $t^{\mu}$ with Newton vector $\nu_{s_{0} s_{1}}=\mu=(2,0)$. Note that in this case, $\tau$ commutes with $s_{0}$ and $s_{1}$.

Accordingly, this case should be added to the table on p. 341. As mentioned above, Thm. 5.1.2 is correct as stated. 


\section{Closure relations}

Denote by $\kappa_{G}: G(L) \rightarrow \pi_{1}(G)_{\Gamma}$ the Kottwitz homomorphism for the group $G$. Here $\pi_{1}(G)$ denotes the algebraic fundamental group of $G$. As usual, we view $\mathbb{J}_{\tau}(F) \subset \mathbb{J}_{\tau}(L)=G(L)$. In the corrected statement of Prop. 7.2.2 below, part (1) remains unchanged. However, since all parahoric subgroups are contained in $\operatorname{Ker} \kappa_{G},(1)$ implies that $\kappa_{G}(j)=\kappa_{G}\left(j^{\prime}\right)$. Since this is not automatic in parts (2) and (3), we have to add this condition there:

Proposition 7.2.2. Let $\Sigma, \Sigma^{\prime} \in \mathcal{I}$ and $j, j^{\prime} \in \mathbb{J}_{\tau}$. The following are equivalent:

1. $j\left(\mathbb{J}_{\tau} \cap P_{\tilde{\mathbb{S}}-\Sigma}\right) \cap j^{\prime}\left(\mathbb{J}_{\tau} \cap P_{\tilde{\mathbb{S}}-\Sigma^{\prime}}\right) \neq \emptyset$,

2. $\kappa_{G}(j)=\kappa_{G}\left(j^{\prime}\right)$ and $j\left(\mathbb{J}_{\tau} \cap P_{\widetilde{\mathbb{S}}-\Sigma}\right) j^{-1} \cap j^{\prime}\left(\mathbb{J}_{\tau} \cap P_{\widetilde{\mathbb{S}}-\Sigma^{\prime}}\right)\left(j^{\prime}\right)^{-1}$ contains a (rational) Iwahori subgroup of $\mathbb{J}_{\tau}$,

3. $\kappa_{G}(j)=\kappa_{G}\left(j^{\prime}\right)$ and the faces in the rational building of $\mathbb{J}_{\tau}$ corresponding to $j\left(\mathbb{J}_{\tau} \cap P_{\widetilde{\mathbb{S}}-\Sigma}\right) j^{-1}$ and $j^{\prime}\left(\mathbb{J}_{\tau} \cap P_{\widetilde{\mathbb{S}}-\Sigma^{\prime}}\right)\left(j^{\prime}\right)^{-1}$ are neighbors (i.e., there exists an alcove which contains both of them).

The proof in loc. cit. proves the corrected statement. In fact, (1) implies $\kappa_{G}(j)=\kappa_{G}\left(j^{\prime}\right)$ as pointed out above. On the other hand, in line 3, p. 348, of loc. cit., we really only get that $\left(g^{\prime}\right)^{-1} j g$ lies in the stabilizer of $I$ in $G(L)$. This implies $\left(g^{\prime}\right)^{-1} j g \in I$ if (and only if) $j \in \operatorname{Ker} \kappa_{G}$.

From the proposition, we get the description in Theorem B above: In fact, it is shown in loc. cit., that the index set in question is $\mathbb{J}_{\tau} /\left(\mathbb{J}_{\tau} \cap P_{\tilde{\mathbb{S}}-\Sigma}\right)$. On the other hand, the group $\mathbb{J}_{\tau} \cap \operatorname{Ker}\left(\kappa_{G}\right)$ acts transitively on the set of simplices of type $\Sigma$ in the Bruhat-Tits building of $\mathbb{J}_{\tau}$ over $F$, and the stabilizer of the face of type $\Sigma$ of the base alcove is $\mathbb{J}_{\tau} \cap P_{\tilde{\mathbb{S}}-\Sigma}$ (but note that the stabilizer of this face inside $\mathbb{J}_{\tau}$ is larger, in general).

\section{Singularities}

The statement of loc. cit., Prop. 7.3.2, while correct, can be generalized and simplified as follows:

Proposition 7.3.2. For all of the tuples $(\tilde{W}, \lambda, J, \sigma)$ given in loc. cit., Section 6.2 (and in the additional case given in Section 2 above),

(1) for all $\Sigma \in \mathcal{I}$, the closure $\overline{Y\left(w_{\Sigma}\right)}$ of $Y\left(w_{\Sigma}\right)$ inside $\mathcal{N}$ has at most isolated singularities, and

(2) the closure $\overline{Y\left(w_{\Sigma}\right)}$ is smooth if and only if $\tau(J) \neq J$ or $\ell\left(w_{\Sigma}\right) \leq 1$. 
The cases where all closures $\overline{Y\left(w_{\Sigma}\right)}$ are smooth are the ones marked (*) in the table in loc. cit., Prop. 7.3.2, and the case described in Section 2 above, i.e.,

$$
\begin{aligned}
& \left(\tilde{A}_{n}, \omega_{1}^{\vee}, \mathbb{S}, \mathrm{id}\right), \quad\left(\tilde{A}_{n}, \omega_{1}^{\vee}, \mathbb{S}, \sigma_{0}\right), \\
& \left(\tilde{A}_{1}, 2 \omega_{1}^{\vee}, \mathbb{S}, \mathrm{id}\right), \quad\left(\tilde{A}_{3}, \omega_{2}^{\vee}, \mathbb{S}, \mathrm{id}\right), \quad\left(\tilde{A}_{3}, \omega_{2}^{\vee}, \mathbb{S}, \sigma_{0}\right), \\
& \left(\tilde{C}_{2}, \omega_{2}^{\vee}, \mathbb{S}, \mathrm{id}\right), \quad\left(\tilde{C}_{2}, \omega_{2}^{\vee}, \tilde{\mathbb{S}}-\{1\}, \mathrm{id}\right) .
\end{aligned}
$$

In fact, the proof given in loc. cit. applies to all those cases. Cf. Wu's paper [3], Rmk. 4.8.

\section{References}

[1] U. Görtz, X. He, Basic loci of Coxeter type in Shimura varieties. Cambridge J. Math. 3 (2015), 323-353.

[2] U. Görtz, X. He, S. Nie, Fully Hodge-Newton decomposable Shimura varieties. Preprint arxiv:1610.05381 (2016).

[3] H. Wu, The supersingular locus of unitary Shimura varieties with exotic good reduction, $\mathrm{PhD}$ thesis, University of Duisburg-Essen, arXiv: 1609.08775 (2016).

ULRICH GÖRTZ

UNIVERSITÄT DUISBURG-ESSEN

FAKULTät FÜR MATHEMATIK

45117 ESSEN

GERMANY

E-mail address: ulrich.goertz@uni-due.de

Xuhua He

UNIVERSITY OF MARYLAND

College Park

MD 20742

USA

E-mail address: xuhuahe@math.umd.edu 